\title{
Mathematical Model for the Homogenization of Unit Load Formation
}

\author{
Béla Illés, Gabriella Bognár \\ Faculty of Mechanical Engineering and Informatics, University of Miskolc, Miskolc, Hungary \\ Email: altilles@uni-miskolc.hu, v.bognar.gabriella@uni-miskolc.hu
}

Received September 2013

\begin{abstract}
One of the most important issues in storage and transport processes is the formation of unit loads. Our main goal is to investigate the homogenization of unit load formation cases. We provide a model involving the major factors and parameters for the optimal selection of the unit load formations. Objective functions and constraints related to the basic tasks are formulated. We give a method for the selection of the optimal unit load formation equipment for a given number of products under given constraints.
\end{abstract}

\section{KEYWORDS}

\section{Unit Load; Unit Load Formation Device; Homogeneous Unit Load; Branch and Bound Method}

\section{Introduction}

In the development of transport, storage and distribution design within supply chain, great emphasis is put on the planning of low task consuming material flows. The fast and efficient way of handling and storing components, raw materials, semi-finished and finished products plays a significant role in this process. Therefore, one of the most important issues in storage and transport processes is the formation of unit loads. It is designed for simplifying the cargo and storage operations as well as for reducing their frequency.

The most common areas applicable on any area of materials handling are the materials handling inside the plant, the materials handling between plants, the in-plant storage, the outside transportation, the commercial storage and the distribution systems [1-6,8].

According to the design of the unit load formation devices the most important devices are

- standardized pallets,

- columnar pallets,

- box pallets,

- platform pallets (wood, metal, wire mesh),

- roller pallets,

- shock absorbent pallets,

- disposable pallets,

- skids,

- storage baskets,

- tote pans,

- containers

The two main tasks during the unit load formation are [7]

1. to choose the proper unit load formation equipment to the goods,

2. to determine the way of loading goods into the unit load formation equipment

The following conditions must be satisfied during the selection of the equipment

1. the goods must fit into the unit load formation equipment,

2. the weight of the goods cannot exceed the carrying capacity of the unit load formation equipment,

3. the position of the goods must be fixed inside the equipment,

4. the unit load formation equipment must fit into the storage areas, loading devices and transporting vehicles 
The unit load types can be classified as

1) Homogeneous (only one type of goods placed in the unit load formation equipment)

2) Non-homogeneous (or mixed when several kinds of goods placed in the equipment)

We review the main factors which appear in the homogenization method of the unit load formation. An optimization process is introduced for the selection of the most appropriate equipments under different constraints. The method is exhibited through the solution to a given example.

\section{Homogenization Process}

Definition: The method of choosing optimal number of unit load formation equipment types subject to given number of goods and maximized number of unit load formation equipments is called the homogenization of the unit load formation equipments. We review four different cases depending on the constraints.

In our investigations the following indices will be applied:

- $\quad$ product identifier: $i=1, \ldots, m$

- $\quad$ equipment model number: $v=1, \ldots, r$

\subsection{No Constraint on the Type of Unit Load Formation Equipments}

Let us define the loading matrix $\mathbf{A}$ by

$$
\left.\mathbf{A}=\begin{array}{c}
1 \\
\vdots \\
i \\
\\
\vdots \\
m
\end{array}\right]
$$

where the number of goods $l_{0}$ is not greater than the type number of unit load formation equipments $r$, i.e.,

$$
l_{0} \geq r .
$$

In this case, we search for the maximal item of each row in the loading matrix and we choose the unit load formation equipment belonging to the column of goods with maximal item.

\subsection{Constraint on the Unit Load Formation Equipment Types}

Let us suppose that

$$
l_{0}<r .
$$

1) If $m \leq l_{0}$, i.e., the product type number is not greater than the equipment type number, then the optimal unit load formation equipment type can be given by choosing a maximal per line item.

2) If $m>l_{0}$, i.e., the product type number is greater than the equipment type number, then

$$
K=\sum_{v=1}^{r} \sum_{i=1}^{m} k_{i v} a_{i v} x_{i v}=\max !,
$$

where $x_{i v}=1$, if the $v$-th equipment is chosen for the $i$-th product, otherwise $x_{i v}=0$, and $k_{i v}$ is the weight factor of the $i$-th product for the $v$-th equipment. A further condition of the model is that for each product exactly one equipment has to be chosen; $x_{i}=\sum_{v=1}^{r} x_{i v}=1$. Let

$$
\beta_{v}=\operatorname{sgn}\left(\sum_{v=1}^{m} x_{i v}\right)
$$

and $\beta_{v}=1$ if the $v$-th equipment is chosen. Therefore the upper bound condition for the unit load formation equipment is

$$
\beta_{0}=\sum_{v=1}^{r} \beta_{v} \leq l_{0}
$$


Summarizing the problem is formulated as:

$$
\begin{gathered}
x_{i} \in\{0,1\} \\
x_{i}=\sum_{v=1}^{r} x_{i v}=1 \\
\sum_{v=1}^{r} \operatorname{sgn}\left(\sum_{v=1}^{m} x_{i v}\right) \leq l_{0} \\
K=\sum_{v=1}^{r} \sum_{i=1}^{m} k_{i v} a_{i v} x_{i v}=\max !
\end{gathered}
$$

This problem can be solved by Branch and Bound Method or dynamic programming.

\subsection{Constraint on the Number of Unit Load Formation Equipments by Type}

If more type of equipments can be applied for a product then let us denote by $\bar{M}_{i}$ the quantity of $i$-th product placed into the equipment. The following conditions has to be met

1) each product has to be placed such that no space is left on either equipment

$$
\sum_{v=1}^{r} a_{i v} x_{i v}=\bar{M}_{i},
$$

2) the number of equipment is the bound:

$$
\sum_{v=1}^{m} x_{i v} \leq c_{v}
$$

3) the product numbers are integers:

$$
x_{i v}=\text { Integer }
$$

The objective function can be formulated as

$$
K=\sum_{v=1}^{r} \sum_{i=1}^{m} k_{i v} a_{i v} x_{i v}=\max !
$$

If there is no solution, the first strong condition can be substituted by a less rigorous one

$$
\sum_{v=1}^{r} a_{i v} x_{i v} \geq \bar{M}_{i} .
$$

If only one type of equipment can be applied for a product then let us define matrix $\mathbf{B}$

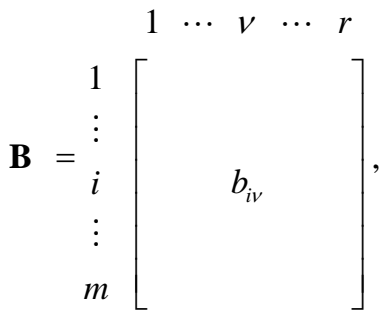

which gives the equipment demand for the average amount of the product. Hence

$$
b_{i v}=\operatorname{Entier}\left(\frac{\bar{M}_{i}}{z_{i v}}+0.5\right),
$$

where $z_{i v}$ denotes the quantity of goods placed on the equipment. The conditions are the following

1) for each product only one equipment can be chosen,

2) $x_{i v}=1$ if the $v$-th equipment is chosen for the $i$-th product, otherwise $x_{i v}=0$,

$$
X_{i}=\sum_{v=1}^{r} X_{i v}=1, \quad X_{i} \in\{0,1\} .
$$


3) The equipment number is the bound

$$
y_{v}=\sum_{v=1}^{m} x_{i v} b_{i v} \leq c_{v}
$$

The objective function can be given by

$$
K=\sum_{v=1}^{r} \sum_{i=1}^{m} k_{i} b_{i v} x_{i v}=\max !,
$$

where the weight factor $k_{i}$ can be chosen as an average quantity: $k_{i}=\bar{M}_{i}$.

The model can be solved by linear programming.

\subsection{Lower Bound on the Number of Equipment Purchases}

Only one equipment can be selected for a product:

1) $x_{i v}=1$ if the $v$-th equipment is chosen otherwise $x_{i v}=0 ; x_{i}=\sum_{v=1}^{r} x_{i v}=1, \quad x_{i} \in\{0,1\}$

2) the bound on the number of equipment $y_{v}=\sum_{v=1}^{m} x_{i v} b_{i v} \geq d_{v}$

The objective function is $K=\sum_{v=1}^{r} \sum_{i=1}^{m} k_{v} b_{i v} x_{i v}=\min$ !

where $k_{v}$ denotes the cost of the equipment. The model is solvable by linear programming.

\section{The Branch and Bound Method}

The selection of the unit load equipment is made by product [3].

1) If the number of product types $m$ is less than the number of the unit load equipments $p$ then $l_{\max }=m$.

2) If $m>p$ then $l_{\max }=m$,

3) If the types of the allowed unit load equipments is $l_{0}$, then $l_{\max }=l_{0}$ :

a) when $l \leq l_{0}$, there is no need to homogenization,

b) when $l>I_{0}$ then the asset diversity must be reduced. The weight factor applied for the selection of the device for the $v$-th equipment and $i$-th product is $k_{i v}$.

In the weighting factor $t_{i v}=\frac{u_{i}}{u_{0}} \frac{x_{i v}}{x_{i}}$,

$\frac{u_{i}}{u_{0}}$ denotes the relative frequency of the $i$-th product, where $u_{0}=\sum_{i=1}^{m} u_{i}$ and $u_{i}=\sum_{\delta=1}^{v} u_{i \delta}$ is the relative frequency of the $v$-th quantity. Let us form the matrices

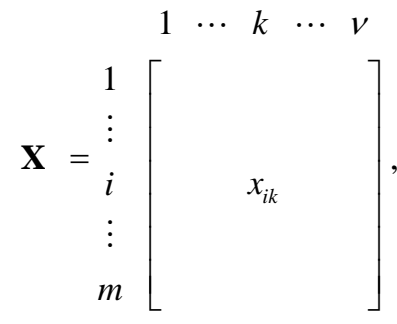

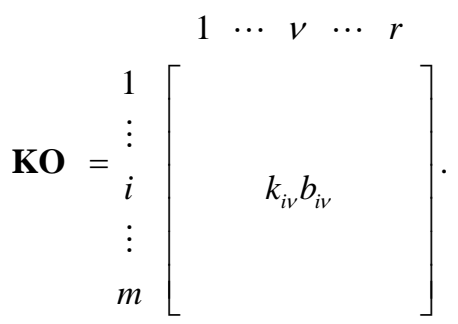


The objective function is

$$
K M=\sum_{v=1}^{p} \sum_{i=1}^{m} k_{i} b_{i v} x_{i v}=\max !
$$

and $x_{i v}=1$ if the $i$-th product is selected for the $v$-th equipment, otherwise $x_{i v}=0, x_{i} \in\{0,1\}$

$$
x_{i}=\sum_{v=1}^{r} x_{i v}=1 .
$$

For the selection of the initial value of $l_{0}$ we form the sums of the matrix column KO

$$
\beta_{v}=\sum_{v=1}^{m} k_{i v} b_{i v},
$$

then we form the equipments in descending order according to $\beta_{v}$.

We introduce a column order investigation. Let $m>p$. It is advisable to take more objective functions into account. We form the efficiency matrix

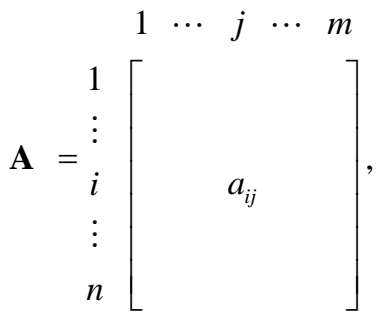

where $a_{i j}=\max \left\{f_{i j \mu}\right\}$ denotes the quantity of the $i$-th product applicable to the $j$-th equipment with the $\mu$-th loading method. The optimal selection is achieved if we find the maximal element in the efficiency matrix $s_{i}=\max _{j}\left\{a_{i j}\right\}$. If we chose the most efficient equipment to each product then the upper bound for the efficiency $s_{0}=\sum_{i=1}^{m} s_{i}$. In order to review the optimal variants we form the inefficiency matrix

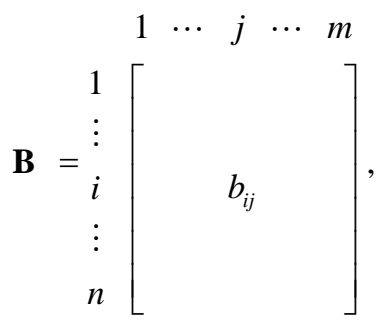

with elements $b_{i j}=s_{i}-a_{i j}$. Let us reduce matrix $\mathbf{B}$. We take the least element in each column $d_{i}=\min \left\{b_{i j}\right\}$. If $d_{j}>0$, then the column can be omitted (as these equipments are not optimal for none of the próducts). If $d_{j}=0$ then the $j$-th column is kept (i.e., it is optimal for at least one product)

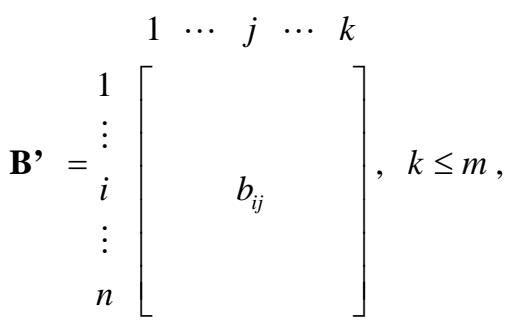

and $k$ denotes the number of column left. Moreover, let $p$ is the number of applicable equipments. If $k \leq p$ then the obtained equipment number is allowed, and the applied number is $k$. If $k<p$, then the equipment numbers have to be reduced. Let us form the sum of the columns: $c_{j}=\sum_{i=1}^{n} b_{i j}$. We sort in order the obtained 
values and take the $p+\delta$ column, where $c_{(p+\delta)^{\prime}}-c_{p^{\prime}}<\varepsilon$ for $\delta$ and $\varepsilon$ is an appropriate small value;

$c_{1^{\prime}} \leq c_{2^{\prime}} \leq \ldots \leq c_{(p+1)^{\prime}} \leq \ldots \leq c_{(p+\delta)^{\prime}}$. On the base of this order we form a possible $p$ column combination from the $p+\delta$-th column of $\mathbf{A}$. Let the $r$-th combination of $\mathbf{A}$ is

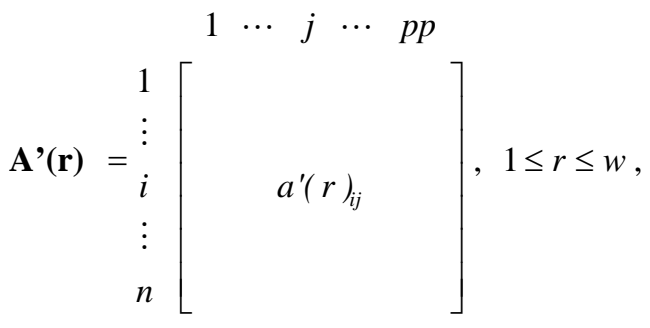

$r=1, \cdots, w$. We form the maximal value of the rows: $s_{i}{ }^{\prime}(r)=\operatorname{ma}_{j}\left\{a_{i j}{ }^{\prime}(r)\right\}$ and determine the resultant efficiency: $s_{0}{ }^{\prime}(r)=\sum_{i=1}^{m} s_{i}{ }^{\prime}(r)$. The optimum version of all the possible variants corresponds to the greatest efficiency: $s_{0}{ }^{\prime \prime}=\operatorname{ma}_{r}\left\{s_{0}{ }^{\prime}(1), s_{0}{ }^{\prime}(2), \cdots, s_{0}{ }^{\prime}(r), \cdots, s_{0}{ }^{\prime}(w)\right\}$.

\section{Example}

Let us select the most appropriate 4 unit load formation equipment from 5 different equipments for 5 different products.

Then $n=5, m=5, p=4$. The efficiency matrix and row maximums can be calculated as

$$
\begin{aligned}
& \begin{array}{lllll}
1 & 2 & 3 & 4 & 5
\end{array}
\end{aligned}
$$

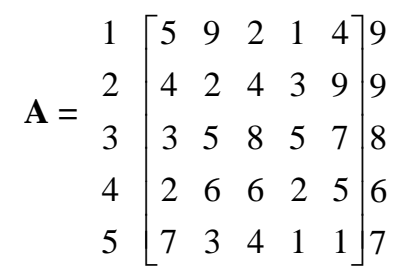

and $s_{0}=\sum_{i=1}^{5} s_{i}=9+9+8+6+7=3$. Let us form the inefficiency matrix by $b_{i j}=s_{i}-a_{i j}$ :

$$
\mathbf{B}=\frac{1}{2}+\left[\begin{array}{lllll}
5 & 9 & 2 & 1 & 4 \\
4 & 2 & 4 & 3 & 9 \\
3 & 5 & 8 & 5 & 7 \\
4 & 6 & 6 & 2 & 5 \\
7 & 3 & 4 & 1 & 1
\end{array}\right]
$$

That gives the values of $d_{i}=\min _{j}\left\{b_{i j}\right\}:\left[\begin{array}{lllll}0 & 0 & 0 & 3 & 0\end{array}\right]$, i.e., the third column can be neglected as it is positive. The reduced $\mathbf{B}$ matrix is

$$
\mathbf{B}^{\prime}=\frac{1}{2} 3\left[\begin{array}{lllll}
1 & 2 & 3 & 4 & 5 \\
3 & 0 & & 7 & 5 \\
5 & 7 & & 5 & 0 \\
5 & 3 & & 0 & 1 \\
4 & 0 & & 0 & 1 \\
0 & 4 & & 3 & 6
\end{array}\right] .
$$


As $k=p$, we found the optimal solution. The optimal selection of the equipments for the given problem can be summarized as follows:

\begin{tabular}{ccc}
\hline Product & & Equipment \\
\hline 1 & $\rightarrow$ & 2 \\
2 & $\rightarrow$ & 5 \\
3 & $\rightarrow$ & 3 \\
4 & $\rightarrow$ & $2-3$ \\
5 & $\rightarrow$ & 1 \\
\hline
\end{tabular}

\section{Acknowledgements}

The research work presented in this paper is based on the results achieved within the TÁMOP-4.2.1.B10/2/KONV-2010-0001 project and carried out as part of the TÁMOP-4.1.1.C-12/1/KONV-2012-0002 "Cooperation between higher education, research institutes and automotive industry" project in the framework of the New Széchenyi Plan. The realization of this project is supported by the Hungarian Government, by the European Union, and co-financed by the European Social Fund.

\section{REFERENCES}

[1] B. Illés and G. Bognár, "Mathematical Modeling of the Unit Load Formation,” Applied Mechanics and Materials, Vol. 309, 2013, pp. 358-365. http://dx.doi.org/10.4028/www.scientific.net/AMM.309.358

[2] B. Illés and G. Bognár, “On the Multi-Level Unit Load Formation Model,” Key Engineering Materials, Vol. 581, 2014, pp. 519-526. http://dx.doi.org/10.4028/www.scientific.net/KEM.581.519

[3] B. Illés and G. Bognár, "A Mathematical Model of the Unit Load Formation,” ASIMMOD Asian Simulation and Modeling Conference Proceedings, 19-21 January 2013, pp. 3-10.

[4] J. Cselényi and B. Illés, “Design and Management of Material Flow Systems I,” Miskolci Egyetemi Kiadó, Miskolc, 2006, pp. $1-384$.

[5] B. Illés, E. Glistau and N. I. C. Machado, “Logistik und Qualitätsmanagement,” Budai Nyomda, Miskolc, 2007, pp. 1-195.

[6] B. Illés, E. Glistau and N. I. C. Machado, “Logistics and Quality Management,” Budai Nyomda, Miskolc, 2007, pp. 1-197.

[7] J. A. Tompkins, J. A. White, Y. Bozer, E. Frazelle, J. Tanchoco and J. Trevino, “Facilities Planning,” John Wiley \& Sons, New York, 1996.

[8] D. R. Delgado Sobrino, O. Moravčík, D. Cagáňová and P. Košt’ál, "Hybrid Iterative Local Search Heuristic with a Multiple Criteria Approach for the Vehicle Routing Problem,” ICMST 2010: 2010 IEEE International Conference on Manufacturing Science and Technology, Malaysia, Kuala Lumpur, 26-28 November 2010, pp. 1-5. 